\title{
Manejo actual de colecciones por ultrasonido endoscópico
}

\author{
Viridiana Oregel-Aguilar*, Gustavo López-Arce y Julio C. Zavala-Castillo \\ Servicio de Endoscopia Gastrointestinal, Hospital General de México Dr. Eduardo Liceaga, Ciudad de México, México
}

\section{Resumen}

Recientemente se han descrito técnicas endoscópicas que son una alternativa para el drenaje de colecciones inflamatorias del páncreas que pueden ser por guiadas por medio del ultrasonido endoscópico y colocación de LAMS. En varios foros internacionales en la UEGW2020 Y DDW 2021, los resultados en cuanto al éxito técnico y eventos adversos del drenaje de las WON guiadas por USE con stents plásticos (DP) o metálicos autoexpandibles totalmente cubiertos (FCSEMS) y LAMS con éxito técnico para todos los stents por arriba del 95\%; éxito clínico mayor del $80 \%$ con DP y mayor del 90\% para los FCSEMS y LAMS. En la mayoría de los estudios se prefiere el drenaje de las colecciones posterior a las 4 semanas, ya que disminuye la morbimortalidad asociadas.

La pancreatitis aguda se puede presentar como pancreatitis edematosa intersticial (IEP) o pancreatitis necrotizante, de acuerdo con la ausencia o presencia de necrosis.

La pancreatitis edematosa intersticial a su vez se divide de acuerdo con el tiempo de evolución: $<4$ semanas en colección líquida aguda peripancreática, $>4$ semanas en pseudoquiste pancreático; la pancreatitis necrotizante en $<4$ semanas en colección necrótica aguda y $>4$ semanas en necrosis amurallada (WON).

La necrosis pancreática se puede presentar en el 5 al $10 \%$ de las pancreatitis agudas, incrementando la morbimortalidad; una de las complicaciones es la necrosis pancreática amurallada (WON) la cual actualmente vemos una evolución en su manejo.

La necrosis pancreática compleja es aquella que presenta restos sólidos necróticos y puede contener aire.

Baron, et al. ${ }^{1}$ realizaron una revisión de expertos por la AGA y la junta de gobierno AGA para evaluar el manejo de la necrosis pancreática; en donde recomiendan el inicio de antibióticos en los casos de necrosis infectada con uso de carbapenémicos, quinolonas y metronidazol; el desbridamiento debe evitarse antes de las 2 semanas, ya que se ha asociado con mayor riesgo de morbimortalidad. En pacientes con pancreatitis necrotizante infectada se recomienda esperar 4 semanas para permitir la formación de una pared. El drenaje percutáneo y endoscópico transmural se puede considerar de primera línea para pacientes con WON.

La ESGE ${ }^{2}$ recomienda el drenaje endoscópico o percutáneo en la (sospecha) necrosis amurallada infectada como primer método de intervención, teniendo en cuenta la ubicación de la necrosis amurallada y la experiencia local. En ausencia de mejoría después del drenaje transmural endoscópico de la necrosis amurallada. Se debe preferir la necrosectomía endoscópica o la cirugía mínimamente invasiva (si ya se ha realizado el drenaje percutáneo) a la cirugía abierta como siguiente paso terapéutico; tomando en cuenta la ubicación de la necrosis amurallada y la experiencia local.

Oblizajek, et al. ${ }^{3}$ realizaron un estudio retrospectivo del 2008-2018 que incluyó 19 pacientes con intervención temprana, encontrando que la indicación más común de intervención fue la infección; la media de tiempo de las colecciones al momento de la intervención endoscópica fue de 23 días, en 11 pacientes se 
realizó necrosectomía concurrente. Los autores concluyeron que la intervención endoscópica es segura entre la $3 .^{a}$ y $4 .^{a}$ semana, cuando está presente una pared formada confirmada por TAC.

Driedger, et al. ${ }^{4}$ realizaron un estudio retrospectivo que incluyó 3 centros con pacientes con necrosis pancreática amurallada (WON), el objetivo fue evaluar la necrosectomía transgástrica quirúrgica (TGN). La decisión de intervención se limitó a pacientes seleccionados de acuerdo con la distribución de la necrosis, si existía mayor componente sólido o líquido, porcentaje de parénquima afectado, si existía infección y la experiencia local de cada institución. Analizaron un total de 178 pacientes con edad media de 51 años, el 64\% fueron hombres y el diámetro de la WON fue de $14 \mathrm{~cm}$. Obtuvieron una morbilidad del $38 \%$ y mortalidad del $2 \%$, y el $91 \%$ tuvieron resolución completa de los síntomas. Los autores concluyeron que la TGN es una opción para el manejo de la WON sintomática, pero se debe hacer una selección adecuada y con seguimiento a largo plazo.

Siddiqui, et al. ${ }^{5}$ realizaron un estudio retrospectivo del 2010 al 2015, que incluyó 313 pacientes, el objetivo fue comparar resultados clínicos y eventos adversos del drenaje de las WON guiadas por USE con stents plásticos (DP), metálicos autoexpandibles totalmente cubiertos (FCSEMS) y LAMS. Se obtuvo como resultado un éxito técnico alto para todos los stents del $98-100 \%$, con resolución del $81 \%$ para DP, del $91 \%$ para los FCSEMS y del $90 \%$ para los LAMS. Estos últimos se asociaron con mayor tasa de sangrado, la oclusión fue menor en los LAMS en comparación con los DP y FCSEMS; prediciendo mayor resolución para las WON y menor requerimiento de intervenciones. Los autores concluyeron que el desbridamiento guiado por USE para WON es mejor con LAMS Y FCSEMSS que los DP.

Park, et al. ${ }^{6}$ realizaron un metaanálisis con 15 estudios que incluyeron un total de 1,746 pacientes que tuvo como objetivo evaluar la eficacia clínica y eventos adversos de los stents para el manejo de colecciones de líquido peripancreático guiados por USE. No se observó diferencia de éxito clínico LAMS $89.3 \%$ vs. DPPS $88.4 \%$ o del LAMS $89.3 \%$ vs. FCSEMS $91.8 \%$, no hubo diferencias significativas en la recurrencia de las colecciones en los diversos grupos; se observó mayor riesgo de sangrado para los LAMS, no existió diferencia para migración entre los grupos comparados. Los autores concluyeron que no se tiene superioridad estadísticamente significativa respecto al éxito clínico para LAMS vs. DPPS, pero sí de los FCSEMS respecto a los DPPS.
Kumta, et al. ${ }^{7}$ evaluaron el uso de los LAMS en el drenaje de colecciones de líquido pancreático; se trató de un estudio multicéntrico que evaluó la seguridad y eficacia de estas. Se incluyó un total de 192 pacientes, con tamaño promedio de la colección de $11.9 \mathrm{~cm}$, la tasa de éxito técnico fue del $92.6 \%$, éxito clínico del 92.6\%; los eventos adversos reportados incluyeron: sangrado $5 \%$, infección $1 \%$ y perforación en $1 \%$; no hubo mortalidad reportada. Los autores concluyen que Ios LAMS son seguros y eficaces.

Fugazza, et al. ${ }^{8}$ evaluaron en un estudio retrospectivo multicéntrico los eventos adversos de las LAMS transgástricas; incluyeron 304 pacientes, con éxito técnico del $98 \%$, la tasa de complicaciones fue del $24.3 \%$; siendo graves en el $6.3 \%$, moderadas en el $68.4 \%$, leves en el $25.3 \%$, presentando sangrado en el $7.2 \%$, migración en el $6.6 \%$, infección en el $6.3 \%$ y oclusión en el $4.6 \%$. Los autores concluyeron que los LAMS son seguros y efectivos, pero deben considerar su uso adecuado, sobre todo en WON.

Maatman, et al. ${ }^{9}$ evaluaron el resultado a corto y largo plazo del desbridamiento transgástrico endoscópico y quirúrgico en pacientes con WON, durante el periodo del 2008 al 2019, con un total de 643 pacientes; de los cuales requirieron intervención el $77 \%$, con intervención transgástrica en el $32 \%$; de estos el $68 \%$ fueron quirúrgicos y el $32 \%$ se atendieron endoscópicamente. Concluyendo que ambas técnicas tienen resolución similar, pero en todos los casos se debe individualizar los pacientes.

Van Santvoort, et al. realizaron un estudio multicéntrico de 88 pacientes con WON para someterlos a necrosectomía abierta; de estos el $69 \%$ requirió manejo quirúrgico y el resto manejo escalonado. La comparativa entre complicaciones para abordaje abierto vs. escalonado: mortalidad del 69 vs. $40 \%$, falla multiorgánica del 40 vs. $12 \%$, insuficiencia exocrina del 33 vs. $7 \%$. Los autores concluyen que un manejo escalonado en comparación con la necrosectomía abierta reduce la mortalidad y complicaciones mayores.

Zhang, et al..$^{10}$ realizaron un estudio retrospectivo, internacional y multicéntrico en el que evaluaron el uso de un novedoso LAMS de $15 \mathrm{~mm}$ de longitud para drenaje de colecciones distantes a la pared gástrica 0 duodenal de 10 a $15 \mathrm{~mm}$. Se incluyeron un total de 35 pacientes, el $74 \%$ tenían WON y el $26 \%$ pseudoquiste pancreático y el $20 \%$ tenían extensión a corredera parietocólica. Se logró un éxito técnico en el $97 \%$, en drenaje transgástrico fue del $94 \%$ y transduodenal en el $6 \%$; la distancia de la pared a la colección fue de $11.8 \mathrm{~mm}$, la duración del procedimiento fue de $25 \mathrm{~min}$. 
Se logró la necrosectomía directa en el $51 \%$, éxito clínico en el 100\%, extracción del LAMS a los 31 días, con recurrencia del $1 \%$. Los autores concluyeron que estos LAMS son seguros y efectivos para el drenaje de colecciones que están a $10015 \mathrm{~mm}$ del transductor del USE.

Alberts, et al. ${ }^{11}$ y Yan, et al. ${ }^{12}$ evaluaron el tiempo óptimo para realizar necrosectomía endoscópica directa (DEN), incluyeron 49 pacientes; concluyendo que la necrosectomía directa posterior a la colocación de LAMS en menos de 30 días no da un resultado desfavorable y que reduce el número de necrosectomías. Sin embargo, Rana, et al..$^{13}$ realizaron un estudio retrospectivo de 170 pacientes; quienes se sometieron a DEN, de estos 34 fueron tempranas y 136 retrasadas, obteniendo como resultado en los primeros peores resultados con aumento de la mortalidad, necesidad de necrosectomía abierta y sangrado.

Shah, et al. ${ }^{14}$ realizaron un estudio en el que participaron 19 expertos, para el manejo endoscópico de colecciones líquidas pancreáticas; observando que la intervención temprana (< 4 semanas después del inicio de la pancreatitis aguda) en comparación con la intervención tardía (> 4 semanas después del inicio de la pancreatitis aguda) es técnicamente factible y eficaz; pero puede estar asociada con una mayor incidencia de eventos adversos, necesidad de cirugía y hospitalización prolongada. El acceso guiado por USE se prefiere al acceso guiado por endoscopia convencional. Los stents plásticos de doble cola de cochino proporcionan drenaje adecuado con un perfil de seguridad aceptable, tanto para pseudoquistes como para WON. Los PS de doble cola de cochino son seguros, económicos y eficaces; las tasas de éxito van del 69.7 al $96.6 \%$, el calibre del stent varía de 7 a $10 \mathrm{~F}$ y el número de stents utilizados varía de 1 a 4 . En la DDW del 2021 Bomman, et al. ${ }^{15}$ destacan un trabajo retrospectivo del 2007 al 2019 en el que compararon la seguridad y eficacia del drenaje temprano < 28 días vs. $>28$ días; en el cual incluyeron 37 pacientes en cada grupo, teniendo como resultado $100 \%$ de éxito técnico, con eventos adversos estadísticamente similares, los de $<28$ días tuvieron una estancia hospitalaria más prolongada de 22 días vs.13 días, así como mayor número de pacientes que requirió $\mathrm{UCl}, 24$ vs. $13.5 \%$. Los autores concluyeron que en caso de realizarse debe ser en un hospital de $3^{\text {er }}$ nivel con gran experiencia. Smanta, et al. ${ }^{16}$ compararon el drenaje endoscópico vs. el percutáneo; se trató de un estudio de cohorte del 2018 al 2020 con un total de 218 pacientes, teniendo como resultado mayor éxito clínico de los colecciones drenadas con USE del $92.1 \%$ vs. percutáneo del $64.6 \%$ al igual que las WON fue más alta del $88.9 \%$ vs. $59.1 \%$. Los autores concluyeron que el éxito clínico fue mayor para los drenajes guiados por USE y con menos eventos adversos.

\section{Conclusiones}

En los últimos años se han documentado avances en el manejo de las colecciones inflamatorias pancreáticas, considerando en la mayoría de las revisiones que el abordaje guiado por USE es el de mejor elección, teniendo una tasa de éxito técnico y clínico similar en los diversos tipos de stents, prefiriendo el drenaje de colecciones posterior a las 4 semanas, ya que disminuye la morbimortalidad asociada.

\section{Financiamiento}

La presente investigación no ha recibido ayudas específicas provenientes de agencias del sector público, sector comercial o entidades sin ánimo de lucro.

\section{Conflicto de intereses}

Los autores declaran no tener conflicto de intereses.

\section{Bibliografía}

1. Baron TH, DiMaio CJ, Wang Andrew Y, Morgan KA. American Gastroenterological Association Clinical Practice Update: Management of Pancreatic Necrosis. Gastroenterology. 2020;158(1):67-75.e1.

2. Arvanitakis M, Dumonceau J-M, Albert J. Endoscopic management of acute necrotizing pancreatitis: European Society of Gastrointestinal Endoscopy (ESGE) evidence-based multidisciplinary guidelines. Endoscopy. 2018;50:524-46.

3. Oblizajek N, Takahashi N, Agayeva S, Bazerbachi F, Chandrasekhara V, Levy M, et al. Outcomes of early endoscopic intervention for pancreatic necrotic collections: a matched case-control study. Gastrointest Endosc. 2020;91(6):1303-9.

4. Driedger M, Zyromski NJ, Visser BC, Jester A, Shuterland FR, Nakeeb A., Surgical transgastric necrosectomy for necrotizing pancreatitis a single-stage procedure for walled-off pancreatic necrosis. Ann Surg. 2020;271(1):163-8.

5. Siddiqui AA, Kowalski TE, Loren DE. Fully covered self-expanding metal stents vs. lumen-apposing fully covered self-expanding metal stent vs. plastic stents for endoscopic drainage of pancreatic walled-off necrosis: clinical outcomes and success. Gastrointest Endosc. 2017;85(4):758-65.

6. Park CH, Park SW, Nam E, Jung JH, Jo JH. Comparative efficacy of stents in endoscopic ultrasonographyguided peripancreatic fluid collection drainage: A systematic review and network meta-analysis. J Gastroenterol Hepatol. 2020;35:941-52.

7. Kumta NA, Tyberg A, Bhagat VH. EUS-guided drainage of pancreatic fluid collections using lumen apposing metal stents: An international, multicenter experience. Dig Liver Dis. 2019;51(11):1557-61.

8. Fugazza A, Sethi A, Trindade AJ, Khashab MA. International multicenter comprehensive analysis of adverse events associated with lumen-apposing metal stent placement for pancreatic fluid collection drainage. Gastrointest Endosc. 2020;91(3):574-83.

9. TK Maatman, BL Bick, EP Ceppa, JM DeWitt. Compararison of operative and endoscopic transgastric debridement for patients with necrotizing pancreatitis. 54 Annual Pancreas Club Meeting. Octuber 22, 2020.

10. van Santvoort HC, Besselink MG, Olaf J. A step-up approach or open necrosectomy for necrotizing pancreatitis. N Engl J Med. 2010;362: 1491-502. 
Endoscopia. 2021;33(Supl 1)

11. Zhang LY, Pawa R, Pawa S, Oleas R, Robles-Medrana C, Obaitan I, et al. ID: 3523063 EUS-guided drainage of non-adherent pancreatic fluid collections using the novel $15 \mathrm{~mm}$ length lumen apposing metal stent. Gastrointest Endosc [Internet]. 2021;93(6 Suppl):AB224-AB225. Disponible en: https://www.giejournal.org/article/S0016-5107(21)00738-0/pdf

12. Yan L, Dargan A, Nieto J, Shariaha RZ, Binmoeller KF, Adler DG. Direct endoscopic necrosectomy at the time of transmural stent placement results in earlier resolution of complex walledoff pancreatic necrosis: Results from a large multicenter United States trial. Endosc Ultrasound. 2019;8(3):172-79.

13. Rana SS, Koushal V, Gupta R, Pandey N. EUS-guided drainage of pancreatic fluid collections during COVID-19 pandemic. Endosc Ultrasound. $2021 ; 10(1): 77-8$
14. Shah R, Basha J, Rana S, Dhir V. Endoscopic management of pancreatic fluid collections: Guidelines of Society of Gastrointestinal Endoscopy of India and Indian EUS Club. Journal of Digestive Endoscopy. 2021;12(1).

15. Bomma S, La Selva D, Larsen M. Safary and outcomes of performing early dual modality drainage ( $<28$ days) of pancreatic necrotic collections-a matched case control study. EUS Poster Session On Sunday, May 23, 2021

16. Samanta J, Dhar J, Muktesh G, Kumar-M P, Gupta P, Agarwala R, et al. ID: 3526758 Endoscopic vs. percutaneous dreanage for the management of infected walled off necrosis: time for paradigm shift? Gastrointest Endosc [Internet]. 2021;93(6 Suppl):AB260-AB261. Disponible en: https://www.giejournal.org/article/S0016-5107(21)00800-2/pdf 